\title{
Peer Reviewers of Nepal Journal of Obstetrics and Gynaecology 2012
}

\author{
Prof. Achala Vaidya \\ Dr. Kusum Thapa \\ Prof. Ashma Rana \\ Dr. Meena Thapa \\ Prof. Chanda Karki \\ Dr. Meera Thapa \\ Prof. Dibya Shree Malla \\ Dr. Nira Singh Shrestha \\ Prof. Dhurba Upreti \\ Prof. Paban Kumar Sharma \\ Prof. Geeta Gurung \\ Dr. Rachana Shah \\ Prof. Gehanath Baral \\ Dr. Rashmi Prasad yadav \\ Prof. Jyoti Sharma \\ Dr. Roshan Thapa Raymajhi \\ Prof. Kundu Yangzom \\ Prof. Meeta Singh \\ Prof. Neelam Pradhan \\ Prof. Pramila Pradhan \\ Prof. Pushpa Chaudhary \\ Prof. Reeta Manandhar \\ Prof. Sanu Maiya Dali \\ Prof. Saraswati M Padhye \\ Prof. Sudha Sharma \\ Prof. Veena Shrivastava \\ Dr. Alka Singh \\ Dr. Anjana Karki \\ Dr. Asha Singh \\ Dr. Bekha Laxmi Manandhar \\ Dr. Ganesh Dangal \\ Prof. Heera Tuladhar \\ Dr. Jageshwor Gautam \\ Dr. Josie Baral \\ Dr. Kesang Diki Bhatso Bista \\ Dr. Kiran Regmi
}

